\title{
THE THIRTIETH ANNUAL MEETING OF THE SOCIETY
}

The thirtieth annual meeting of the American Mathematical Society was held at Columbia University on Thursday and Friday, December 27-28, 1923. Dormitory accommodations were furnished by Columbia University and Barnard College, and attending members were introduced at the Faculty Club. About seventy-five members attended the dinner on Thursday evening, at which Professor Coolidge spoke on the progress of the Endowment Fund. At the close of the sessions it was voted to express the thanks of the Society to Columbia University for its courtesy, continued through more than thirty years. It was voted to thank also the Governors of the Faculty Club, and the Committee on Arrangements for the meeting, which consisted of Professors T. S. Fiske (chairman), G. W. Mullins, and L. P. Siceloff, Dr. G. A. Pfeiffer, and Mr. A. E. Meder.

The attendance included the following ninety-five members of the Society:

C. R. Adams, Alexander, C. R. Ballantine, J. P. Ballantine, Barnum, Bernstein, Birkhoff, Borden, R. W. Burgess, Burwell, Clements, Abraham Cohen, Coolidge, C. F. Craig, Dadourian, Douglas, Edmondson, Eisenhart, Fields, Fine, Fite, Forsyth, Philip Franklin, Fry, Gafafer, Gilman, Glenn, P. H. Graham, Grant, M. C. Graustein, W. C. Graustein, Hammond, W. L. Hart, Harter, Hawkes, Hazlett, Robert Henderson, Hille, Himwich, Hotelling, Huntington, Joffe, Kasner, Kazarinoff, Lamson, Langman, Leib, Linehan, Lynch, Mc Giffert, Mac Inness, Mathews, Meder, Mirick, H. H. Mitchell, Frank Morley, Marston Morse, Mullins, Oglesby, Olds, F. W. Owens, Pell, Post, Rainich, Ranum, Raynor, Reddick, R. G. D. Richardson, Ritt, Seely, Seidlin, Shook, Siceloff, Simmons, Slotnick, Simons, C. E. Smith, D. E. Smith, W. M. Smith, Virgil Snyder, M. H. Stone, Elijah Swift, J.S. Taylor, Tracey, Tyler, Veblen, Evelyn Walker, J. L. Walsh, Wedderburn, Weisner, Weiss, H. S. White, E. E. Whitford, Whittemore, R. G. Wood.

The Council elected the following sixty-five persons to membership in the Society:

Professor Olafur T. Anderson, Wesley College;

Professor William Elijah Anderson, Miami University;

Dr. Bernardo Ig. Baidaff, Buenos Aires;

Dr. John Perry Ballantine, Columbia University;

Professor Maurice Baudin, Miami University; 
Professor James Marcus Bledsoe, East Texas State Teachers College; Mr. William Miller Bond, College of the City of New York;

Mr. Orley Edward Brown, Northwestern University;

Dr. Keivin Burns, Allegheny Observatory;

Mr. William Elmer Byrne, Rensselaer Polytechnic Institute;

Professor Theodosia Tucker Calloway, Stephens Junior College;

Professor George B. Clinkscales, Converse College;

Dean James Frost Cross, St. John's College;

Professor Samuel Ridgely Cruse, University of Arizona;

Dr. Heber Doust Curtis, Allegheny Observatory;

Mr. Alfred Davis, Soldan High School, St. Louis;

Mr. Hannibal A. Davis, West Virginia University;

Professor Samuel Dickstein, University of Warsaw;

Mr. Walter Anthony Dynan, Amityville, N. Y.;

Mr. George Gilbert Entz, Los Angeles;

Mr. Elgin George Fassel, Cleveland Life Insurance Company;

Mr. James Hampton Fithian, Brown University;

Mr. Anselm J. Fleisig, St. Procopius College;

Professor Robert Dale Ford, St. Lawrence University;

Miss Alice Ann Grant, Brown University;

Professor Ruby Mabel Grimes, Northern Normal and Industrial School, Aberdeen, S. D.;

Mr. Duncan Claire Harkins, West Virginia University;

Professor Eli Stuart Haynes, University of Missouri;

Professor Chester Rodney Hillard, College of the Ozarks;

Professor Frederick Chapman Jonah, Acadia University;

Mr. Arthur Daniel Kinsman, Ipswich, Mass.;

Professor Carl Dewey Laws, Hampden-Sidney College;

Dean Thomas Jefferson Leslie, Austin College;

Mr. Locke Ellsworth Lunn, State High School, Heron Lake, Minn.;

Mr. Arthur Louis McCarty, Lowell High School, San Francisco;

Dean Lee Marcus McCoy, Morgan College;

Professor Raymond Earl Manchester, Kent State College;

Mr. Alfred Joseph Maria, Rice Institute;

Professor GertrudeWhittier Mendenhall, North Carolina CollegeforWomen;

Mr. Alfred William Muldrew, St. John's Technical High School, Winnipeg;

Mr. James A. Nyswander, University of Chicago ;

Dr. Robert Bowie Owens, Franklin Institute;

Mr. George Ambrose Parkinson, University of Wisconsin;

Professor Frederick Malling Pedersen, College of the City of New York;

Miss Sallie E. Pero, American Telephone and Telegraph Company;

Professor Otto J. Ramler, Catholic University;

Professor James Nelson Rice, Catholic University;

Mr. Harold E. Riter, Daniel McIntyre Collegiate Institute, Winnipeg;

Mr. William Haldeman Rittenhouse, Philadelphia;

Miss Georgia Edna Robinson, Stephens College;

Mr. Harry Mance Roeser, Bureau of Standards ;

Mr. James Shohat, University of Chicago;

Dr. Charles Albert Shook, Yale University;

Professor Robert Frederic Smith, College of the City of New York;

Dean Julia Spalding, Christian College;

Mr. Arthur A. Sperling, University of Wisconsin, Milwaukee Branch;

Mr. Robert Meldrum Stewart, Dominon Observatory, Ottawa;

Professor Robert Ambrose Thornton, Shaw University;

Professor Ernest R. Tucker, Texas Christian University; 
Professor Charles Ambrose Van Velzer, Carthage College;

Mr. Albert Huntoon Wait, University of Wisconsin;

Miss Fannie Watkins, Winthrop College;

Mr. Lawrence E. Widmark, Star Electric Motor Company;

Mr. Edgar William Woolard, United States Weather Bureau;

Mr. Floyd Eugene Young, Oregon Agricultural College.

The total membership of the Society is now 1250, including 83 life members. The total attendance of members at all meetings, including sectional meetings, during the past year was 541; the number of papers read was 266 . The number of members attending at least one meeting was 331. At the annual election 172 votes were cast.

The report of the Treasurer was received, showing a balance of $\$ 9,638.78$, exclusive of special funds; of this, $\$ 5,726.91$ is reserved to secure the life memberships. Sales of the Society's publications during the year amounted to $\$ 3,570.03$. The report of the auditors (Mr. S. A. Joffe and Professor W.J. Berry) was deferred until the February meeting.

The Board of Trustees adopted a budget for 1924 .

The librarian reported that the Library of the Society now contains 6,546 volumes.

At the annual election, which closed on Thursday afternoon, the following trustees and officers and other members of the Council were chosen:

Trustees, Professor G. A. Bliss, Professor W. B. Fite, Mr. Robert Henderson, Professor R. G. D. Richardson, Professor OswaLd VeBlen.

Vice-Presidents, Professor E. V. Huntington (one year); Professors T. H. Hildebrandt and J. H. M. Wedderburn (two years).

Secretary, Professor R. G. D. Richardson.

Assistant Secretary, Professor ARnold Dresden.

Treasurer, Professor W. B. Fite.

Librarian, Professor R. C. ARCHibald.

Member of the Editorial Committee of the Bulletin, Professor ARNold DRESDEN.

Member of the Editorial Committee of the Transactions, Professor EDward Kasner. 
Members of the Council, Professor H.W.TyLER (one year); Professor J. L. Coolidge (two years); Professors HarRY Bateman, G. A. Bliss, Solomon Lefschetz, C. L. E. Moore, Anna J. Pell (three years).

The tellers appointed by President Veblen to count the ballots were Professor R. E. Gilman, Dr. Jesse Douglas, and Mr. A. E. Meder.

An account of the proceedings connected with the presentation of the first Bôcher Memorial Prize and of the regulations governing the second and third awards is contained elsewhere in this issue.

On account of proposed absence from the country, Professor E. B. Van Vleck presented his resignation as representative of the Society on the National Research Council. Professor H. F. Blichfeldt was selected to complete the term which expires on July 1, 1924, and also to represent the Society for the succeeding period of three years. Professors A. B. Coble, Arnold Dresden, L. E. Dickson, and Virgil Snyder were elected as additional representatives of the Society on the American Section of the International Mathematical Union.

A committee on the western meetings of the Society was appointed for a two-year period, consisting of Professors T. H. Hildebrandt (chairman), Henry Blumberg, and Arnold Dresden. Dean H. L. Hodgkins and Professor R. G. D. Richardson were appointed to represent the Society on the Council of the American Association for the Advancement of Science for the year 1924.

The committee on membership was continued, to consist of Professors Abraham Cohen (chairman), E. B. Stouffer, and C. E. Smith (permanent secretary), and such others as President Veblen may appoint.

A letter was read from the secretary of the PhysicoMathematical Society of Japan, thanking the Society for the message of sympathy sent in September and recounting some of the effects of the disaster on mathematical research in that country. 
The Council recommended to the Society that the ByLaws be amended so as to include ex-Presidents in the Council for a period of six years after expiration of their presidential terms.

It was announced that Professor H. H. Mitchell had been appointed chairman of the Committee on the next Colloquium, in place of Professor L. E. Dickson resigned; and that Dean H. L. Hodgkins had represented the Society at the inauguration of President Lewis of George Washington University.

Announcement was made by President Veblen, who is also Chairman of the Division of Physical Sciences of the National Research Council, concerning the extension of one group of the Research Fellowships to include mathematics. This group of fellowships which is adminstered by the Research Fellowship Board of the National Research Council and supported by the Rockefeller Foundation now covers mathematics, physics, and chemistry; and, beginning July 1 , 1925 , the annual income is increased to $\$ 125,000$. In order that adequate use be made of this remarkable addition to the resources for mathematical research, members are urged to give information concerning talented and well-equipped young men and women.

President Veblen presided at the sessions on Thursday, relieved by Vice-President Tyler; during the sessions on Friday, Vice-Presidents Huntington and Wedderburn alternated in the chair.

At the session on Friday morning a paper was read, at the request of the program committee, by Professor Virgil Snyder, on Problems connected with involutorial transformations in space. This paper appeared in full in the March-April issue of this BuLlETIN.

Titles and abstracts of the papers read at this meeting follow below. The papers of Professor Bell, Dr. Zeldin, Professor Lipka, Dr. Pfeiffer, Dr. Wiener, Professor Ford, Mr. Michal, and Professor Ritt, and the second papers of Professor Huntington, Dr. Walsh, Dr. Ballantine, and Dr. Taylor, were read by title. 
1. Professor W. B. Fite: The analytic functions defined by a certain functional differential equation.

In this paper the author considers the solutions in the complex plane of the functional differential equation $y^{\prime \prime}(z)$ $=A^{2} y(\sin z)=0$. He finds that they are entire functions of $z$. Some theorems relating to the existence and distribution of the real zeros of these functions are proved. Every such function has at least one real zero. Nothing is determined concerning the complex zeros.

2. Dr. K. W. Lamson: The field equations of relativity as integrability conditions.

The condition for the vanishing of the sum of the Riemann (two-dimensional) curvatures determined by $\dot{\mu}$ mutually perpendicular directions in the generalRiemann $n$-space is $K_{\mu}=0$, where $K_{\mu}=\sum g_{i j} g_{i j} R_{i i},{ }_{j j}$. Here $R_{i i, j, j}$ is the RiemannChristoffel 4-index symbol, and the $i$ 's are any $\mu$ numbers from the set $1,2, \ldots, n$, and similarly for the $j$ 's. The summation is taken over all permutations of the $i$ 's among themselves and of the $j$ 's among themselves. The object of the paper is to prove that $K_{\mu}=0$ is a necessary and sufficient condition for the existence of a contact transformation which transforms $\sum g^{i j} p_{i} p_{j}$ into the sum of squares. This contact transformation is determined by $n-\mu+2$ relations between the old and new point coordinates. In particular, if $n=4$, then $K_{3}=0$ is equivalent to the Einstein field equations. The general solution of the field equations is found by algebraic processes.

3. Dr. Jesse Douglas: Characterization of spaces of constant curvature by the arrangement of their geodesics.

Necessary conditions are derived that a family of curves in $n$-space defined by a system of differential equations of the form $d^{2} x_{i} / d x_{1}^{2}=G_{i}\left(x_{j}, d x_{j} / d x_{1}\right)$ be such that through every three points a two-spread passes which contains $\infty^{2}$ curves of the family. As a special case, a proof is obtained of Schur's theorem (Mathematische ANNALEn, 1886) that the existence of a geodesic two-spread through every three points of a Riemann space characterizes the space as having constant curvature. A generalization is developed to the effect that "two-spread" can be replaced by " $k$-spread". 
4. Mr. G. Y. Rainich: The non-linearity of Maxwell's equations in general relativity theory.

The Maxwell equations consist of two sets of four equations each which have to be satisfied, respectively, by the covariant and mixed components of the electromagnetic tensor; for vacuum each set is linear. But these two sets are not sufficient to determine the electromagnetic field; we must consider together with them the equations which give the connection between the covariant and mixed components, and these equations involve the $g$ 's which are connected with the coefficients of the electromagnetic tensor by the energy relation which is not linear; therefore the whole system is non-linear. In order to be able to consider these things without introducing the restriction connected with the use of the $g$ 's, we introduce a new system of representation for curved space*. Each point is characterized by four coordinates as usual, but to represent vectors and tensors we introduce in every bundle cartesian coordinates. We have thus no distinction between coand contravariant quantities, and the algebra of tensors is simplified. But additional complications arise in the tensor analysis, and they are responsible in this case for the non-linearity of Maxwell's equations.

5. Professor J. L. Coolidge: A simple proof of Lüroth's theorem.

Lüroth's theorem states that if $x$ and $y$ are rational functions of a parameter $t$, not both constants, the curve so defined has the deficiency zero. The author gives a new proof based on Zeuthen's correspondence formula, and communicates one by Professor Osgood based on abelian integrals.

6. Professor B. A. Bernstein: Representation of threeelement algebras.

The author gives a method of obtaining a concrete representation of any binary operation or dyadic relation possible in an algebra consisting of three elements. The representations are all arithmetic and modular. The device used is an extension of that employed by him in the representation of two-element algebras.

* Cf. the author's paper in Proceedings of the National Academy, Dec., 1923. 
7. Dr.J.L.Walsh: On the expansion of analytic functions in series of polynomials.

Let $C$ be a simple closed finite analytic curve in the $z$-plane including in its interior the origin. Then there exist two sets of functions, $p_{0}(z), p_{1}(z), \ldots, p_{n}(z), \ldots$ and $q_{1}(z), q_{2}(z), \ldots, q_{n}(z), \ldots$, polynomials respectively in $z$ and in $1 / z$, such that if $f(z)$ be any function defined on $C$ and satisfying on $C$ a Lipschitz condition, then $f(z)$ can be expanded in the form $f(z)=a_{0} p_{0}(z)+a_{1} p_{1}(z)+\cdots+a_{n} p_{n}(z)$ $+\cdots+b_{1} q_{1}(z)+b_{2} q_{2}(z)+\cdots+b_{n} q_{n}(z)+\cdots$, where the former series converges uniformly in the closed region interior to $C$ and the latter series converges uniformly in the closed region exterior to $C$ and vanishes at infinity. The coefficients are given by formulas $a_{k}=\int_{C} f(z) s_{k}(z) d z$, $b_{k}=\int_{C} f(z) t_{k}(z) d z$, where the functions $s_{k}(z)$ and $t_{k}(z)$ depend on $C$ but not on $f(z)$. The polynomial $p_{k}(z)$ has precisely $k$ roots interior to $C$, and the polynomial $q_{k}(z)$ has precisely $k$ roots exterior to $C$.

8. Professor E. V. Huntington: A new set of postulates for betweenness, with proof of complete independence.

A paper published in 1917 by E.V.Huntington and J.R.Kline (Transactions of this Society, vol. 18, pp. 301-325) gave eleven sets of independent postulates for betweenness, selected from a basic list of twelve postulates, A, B, C, D, 1-8. Of these eleven sets, three contained seven postulates each and eight contained six postulates each. In the present paper, a new postulate is added to the basic list, namely Postulate 9 ; it is then shown that the following set containing only five postulates, A, B, C, D, 9, is sufficient to define betweenness:

(A) If $A B C$, then $C B A$.

(B) If $A, B, C$ are distinct, then at least one of the six permutations, $A B C, A C B, B A C, B C A, C A B, C B A$, is a true order.

(C) If $A B C$ is true, then $A C B$ is false.

(D) If $A B C$ is true, then $A, B, C$ are distinct.

(9) If $A, B, C, X$ are distinct and $A B C$ is true, then at least one of the orders $A B X, X B C$ is true.

These five postulates are shown to be completely independent; and the long list of theorems in the earlier paper is extended so as to cover all the inter-relations among the thirteen postulates of the enlarged basic list. The paper will be offered to the Transactions. 
9. Professor E. V. Huntington: A new set of completely independent postulates for cyclic order.

If Postulate A for betweenness (see preceding abstract) is replaced by Postulate $\mathrm{A}^{\prime}$ : If $A B C$, then $B C A$; then the five postulates $\mathrm{A}^{\prime}$, B, C, D, 9 will form a completely independent set of postulates for cyclic order. This paper is supplementary to two earlier papers on cyclic order, one presented on December 28, 1916 (see an abstract in the ProceEDINGS of the National AGademy, vol. 2 (1916), pp. 630-631), and the other on October 27,1923. It will be offered to the Transactions.

10. Professor E. T. Bell: Representations of integers in certain quadratic forms in 2, 3, 4, 5 indeterminates and allied class-number relations.

When there is but one class in the principal genus of binary quadratic forms of a negative determinant, the number of representations of an integer in the form can be found at once in terms of the devisors of the integer. For certain ternary and quaternary forms there are similar simple theorems. For quinary forms the like exists in several cases if the integer represented is the square of an odd integer times a power of 2 . All of the results give class number relations; those relating to quinary forms are of a wholly new type.

11. Dr. J. L. Walsh: On Pellet's theorem concerning the roots of an algebraic equation.

This paper furnishes a complete answer to the following question: If there are given the absolute values of the coefficients of an algebraic equation, what can be said of the regions of the complex plane in which the roots of the equation do or do not lie? The only statement which can be made regarding the roots is essentially a theorem proved by Pellet, Darboux's Bulletin, (2), vol. 5 (1881).

12. Dr. E. L. Post: The mth derivative of a function of a function; calculus of mth derivatives.

At the October, 1923, meeting of the Society the author presented a paper on a theory of generalized differentiation. The application of this to the Heaviside theory requires a usable knowledge of $m$ th derivatives. The problem of 
finding the $m$ th derivative of a function of a function, $F(u)$, has always presented great difficulties. Thus, although the Faa di Bruno formula gives $\left(d^{m} / d x^{m}\right) F(u)$ as a finite series in $F^{(m)}(u)$ with coefficients depending wholly on $u$ and its derivatives, the formula for these coefficients is quite unmanageable. The present paper concerns itself specifically with these coefficients, and sets up formulas for the coefficients of a sum, product, and quotient of two functions, and for a function of a function in terms of the coefficients of these functions. Thus in the last case if $v$ is a function of $u$ and $u$ a function of $x$,

$$
(m) v /(n) x=\sum_{l=m}^{n}((m) v /(l) u)((l) u /(n) x),
$$

where $(m) u /(n) x$ is the $m$ th coefficient in the $n$th derivative of a function of $u$. These rules in connection with the coefficients for the simplest elementary functions may be said to constitute a calculus of the coefficients in question, and this in turn, added to the previously known formulas, gives a calculus of $m$ th derivatives.

13. Professor W. C. Graustein: Invariant directions in the theory of surfaces.

Given a single surface or a map of one surface upon another, there exist directions, or vectors, which are relatively invariant under a change of parameters. It is the purpose of this paper to determine directions with this property and to demonstrate their usefulness in the theory of surfaces and the theory of maps.

14. Mr. D. K. Kazarinoff: A property of the curvature of the line of intersection of two surfaces.

Consider the two surfaces $\sigma_{1}$ and $\sigma_{2}$ and any point $P$ of their line of intersection $\left(\sigma_{1}, \sigma_{2}\right)$, where $P$ is not a singular point for either of the surfaces. Consider at $P$ the two planes $\tau_{1}$ and $\tau_{2}$ tangent to $\sigma_{1}$ and $\sigma_{2}$ respectively, and the plane curves of intersections $\left(\sigma_{1}, \tau_{2}\right)$ and $\left(\sigma_{2}, \tau_{1}\right)$. We regard the curvature of a curve as a vector of length equal to the absolute value of the curvature and directed as the principal normal from the curve to its center of curvature. We have, then, the following theorem, believed to be new: The curvature of $\left(\sigma_{1}, \sigma_{2}\right)$ at $P$ is the geometric sum of the curvatures of $\left(\sigma_{1}, \tau_{2}\right)$ and $\left(\sigma_{2}, \tau_{1}\right)$. A proof of remarkable simplicity, based on Meusnier's theorem, can be given. 
15. Dr. Louis Weisner: Simultaneous algebraic equations.

Let two plane algebraic curves whose equations have their coefficients in a domain $R$ intersect in a finite number of points all of which are in the finite part of the plane. It is shown that the group relative to $R$ of the equation which the abscissas of the points of intersection of the two curves satisfy is the same as that which the ordinates satisfy. A similar theorem holds for any finite number of algebraic equations which are independent and consistent.

16. Professor Virgil Snyder: On the types of monoidal involutions.

The purpose of this paper is to unify the theory of monoidal involutions by proving all the known theorems by means of the same method. A number of new types are discussed, and it is shown that if irrational types exist they must be compounded from the perspective monoidal types, either by means of the plane Bertini type or the perspective Jonquières type. The involution which belongs to the cubic variety of four-dimensional space is included in the latter category.

17. Dr. Einar Hille: On the zeros of the functions of the parabolic cylinder.

The differential equation of the parabolic cylinder can be brought to one of the two forms $w^{\prime \prime} \pm\left(z^{2}-a^{2}\right) w=0$, where $a$ is real in general. The paper contains a study of the set of zeros and extrema of a given solution of one of these differential equations. The problem is considered from three different angles: (1) the asymptotic distribution of the set, (2) the geometric structure of the set, and (3) the variation of the structure with different initial conditions and with the parameter $a$.

18. Professor Edward Kasner: Geodesic families and their generalizations.

In this paper the author discusses various generalizations of the family of straight lines in ordinary $n$-space or geodesics in a Riemann space, in particular cubic families, extremal families, and intersectional families. Cubic families (which include the natural and velocity types previously studied by the author) are related to the geodesics of 
Weyl's general affine connected space or the path geometry of Eisenhart and Veblen. Extremal families are related to the general integral of first order in the calculus of variations. Intersectional families are defined by the curves of intersection of families of surfaces or hypersurfaces (just as straight lines are obtained from planes). The overlapping of the various types is considered.

19. Dr. J. S. Taylor: A statistical theory of depreciation. The principal results obtained are, first, a means of determining more accurately than heretofore the useful life of the average machine of a given type; second, a method of distributing the depreciation charges in such a way that the unit cost of output remains constant (or increases by given amounts); and third, a method of determining when an individual machine should be discarded when that time arrives in which the only conjecture is in the estimates of the costs for a new machine. Finally, the paper points out the interdependence of costs of output and the distribution of the depreciation charges, together with the fact that the useful life of a machine is affected by such distribution and by the current interest rate.

20. Dr. S. D. Zeldin: A classification of integral invariants in the calculus of variations.

The curve $y=f(x)$ which maximizes or minimizes the integral $I=\int F\left(x, y, y^{\prime}, y^{\prime \prime}, \ldots, y^{(n)}\right) d x$ satisfies Lagrange's equation. The integral $I$ is invariant under point and contact transformations. In this paper the author classifies the integrals $I$ invariant under the contact transformations of the plane.

21. Professor Virgil Snyder: Problems connected with involutorial transformations in space.

This paper appeared in the March-April issue of this BULLETIN.

22. Professor Joseph Lipka: Some theorems on trajectories in $V_{n}$.

A study is made of a system of trajectories in a conservative field of force in space of $n$ dimensions, taking, as a basis, $n$ mutually orthogonal congruences and the Ricci coefficients of rotation $\gamma_{\alpha \beta h}$. Among other theorems, 
the author proves that the sum of the squares of the geodesic curvatures of any $n$ mutually orthogonal trajectories through a point is an invariant, $i$. e., independent of the $n$ directions chosen; this invariant is equal to $(n-1)$ times the square of the magnitude of the trajectory vector at the point.

23. Professor Joseph Lipka: On irreversible dynamical systems under a zero force.

These curves may be defined as the extremals of the variation problem $\int d s+\int d \Phi=$ minimum, where $d s^{2}$ is the fundamental quadratic differential form in the space, and $d \Phi=\sum \Phi_{i} d x_{i}$, the $\Phi_{i}$ being functions of the coordinates. An interesting geometric property of these curves is the following: The locus of the centers of geodesic curvature of the $\infty^{1}$ curves of the system which pass through a given point is a hyperplane if the dimensionality of the space is odd; on the other hand, the above locus is a quartic hypersurface of a special variety if the dimensionality of the space is even. A curve of the system defined above may also be identified with Weyl's "SingularitätsKanal" in a field with a given structure.

24. Professor Marston Morse: Relations between different types of critical points of a real analytic function of $n$ real variables.

If there be given a real analytic function of $n$ variables, Birkhoff has shown that, under certain boundary hypotheses, the existence of $m$ proper minima $(m>1)$ implies the existence of other critical points affording neither maxima nor minima. The present paper attacks the problem of determining the relations between general types of critical points each characterized by the signature of the quadratic form making up the terms of second order in the expansion of the given function in the neighborhood of the given critical point.

25. Dr. C. R. Adams: The general theory of the linear $q$-difference equation.

The author uses matrix methods in treating the system

$$
g_{i}(q x, r y)=\sum_{j=1}^{n} p_{i j}(x, y) g_{j}(x, y), \quad(i=1,2, \ldots, n),
$$


in which $q$ and $r$ are real or complex constants different from zero, the $p_{i j}(x, y)$ are polynomials in $x$ and $y$, and the $g_{i}(x, y)$ are the functions to be determined; such a system is equivalent to a single homogeneous equation of the $n$th order. If $|q| \lessgtr 1,|r| \lessgtr 1$, the system possesses in general four matrices of formal series solutions (in powers of $x$ and $y, 1 / x$ and $1 / y, x$ and $1 / y, 1 / x$ and $y$ ). The case $|q|,|r|>1$ is typical; then the elements of two matrices converge to analytic functions in the vicinities respectively of $(0,0)$ and $(\infty, \infty)$. Two matrices of solutions are thus defined, one analytic whenever neither $x$ nor $y$ is infinite, and the other analytic save for poles whenever neither $x$ nor $y$ is zero. A matrix of periodic functions is defined by these two solutions, which, after transformation, appears as a matrix of triply periodic functions, a special case of a class of such functions discussed by Cousin. An inverse of the general existence theorem is proved and a method given for handling certain exceptional cases. It is further proved that if $|q|=|r|=1$, there exists in general no analytic solution.

26. Professor O. F. Glenn: The invariants of forms under the total group $G_{6}$, modulo 2.

This paper appeared in full in the March-April number of this BULLETIN.

27. Professor Olive C. Hazlett: On associated forms in the theory of formal modular covariants.

By the aid of the symbolic theory of formal modular covariants, there is proved for such covariants a theorem which is closely analogous to Hermite's well known results for algebraic covariants. If $h$ be any formal modular covariant of the binary form $f$ with respect to the Galois field $G F\left[p^{n}\right]$ of order $p^{n}$, then (aside from a power of $h$ ) every formal modular covariant of $f$ is expressible as a polynomial in the associated covariants with respect to $h$ and a finite number of formal modular covariants obtained from $h$ in a certain manner.

28. Professor Edward Kasner: Intermediate curvatures in a Riemann space.

A result analogous to Schur's theorem on Riemann curvature holds for Ricci curvature and for the intermediate 
curvatures defined by the author in a previous paper: If any one of the curvatures is a point function (instead of an orientation function) it must be a constant. When this is proved for the Ricci curvature (by making use of Hilbert's identities) Schur's theorem is obtained as an immediate corollary, and by combining these results in a simple manner suggested by Mr. Rainich, the general theorem is obtained. When the curvatures are equated to zero, we obtain spaces which Dr. Lamson has integrated by means of contact transformations.

29. Dr. G. A.Pfeiffer: A proof of Cauchy's integral theorem for any rectifiable boundary.

Cauchy's integral theorem in the strong form, namely, if $f(z)$ is analytic in a domain bounded by any rectifiable curve and continuous on the set consisting of that domain and its boundary, then the integral of $f(z)$ over the boundary is zero, was established, so it seems, only recently. E. B. Van Vleck published in 1921 a proof of Green's lemma for any rectifiable boundary. The above theorem follows in the familiar way from that result. A direct proof of said theorem was published this year by S. Pollard, who in deriving certain preliminary results of a semi analysis situs character made use of some special results of de la Vallée Poussin. The present paper gives a proof of the same theorem, based only on well known mathematical facts. The lemma of the paper seems of interest on its own account. It states that the perimeters of all polygons which are within a domain $S$, bounded by a rectifiable curve, and which are derived from rectangular networks in a well known way, are less than $2 \sqrt{2}$ times the length of the boundary of $S$.

30. Dr. J. P. Ballantine: A generalization of the calculus of finite differences to include the differential calculus.

In the calculus of finite differences, one speaks of a difference quotient taken at a set of distinct values. Differential calculus is often regarded as the limiting case when all the values approach equality. In this paper, it is shown that by properly enlarging the notion of difference quotient, differential calculus becomes a special, and not a limiting, case. Other special cases are found to be interesting, and have a practical application to the numerical solution of differential equations. 
31. Dr. J. P. Ballantine: A postulational introduction to the positive integers.

Certain properties of the numbers 0 and 1 , expressible without the use of any other numbers, are supposed. A class of undefined elements, called integers, is postulated, subject to a binary relation, called successor. The successor relation is in the form of a matrix, $\varkappa^{\prime \prime}$, whose values are 0 and 1 according as the second argument is not or is a successor of the first. It is subject to three axioms: Axiom 1, $S^{\prime}\left(1^{\prime}-S^{\prime} \varkappa^{\prime \prime} 1^{\prime}\right) 1^{\prime}=0 ;$ Axiom $2, S^{\prime}\left(1^{\prime}-S^{\prime} 1^{\prime} x^{\prime \prime}\right) 1^{\prime}$ $=1 ;$ Axiom $\left.3, S^{\prime} \xi^{\prime} x^{\prime \prime}=\xi^{\prime} \cdot\right) \cdot \xi^{\prime}=0^{\prime}$. It is proved that the successor of each integer is unique, that there exists (uniquely) a first integer, 1, that the set of successive successors of the integer 1 includes all integers except 1 , and that the integers are linearly ordered.

32. Dr. J. S. Taylor: A note on the theory of testimony.

This paper deals with the question of determining the probability of the occurrence of a given event, whose a priori probability is known, when attested to by one or more observers. The personal accuracy of observation of an individual observer is defined as the limit of the accuracy of his observation as the a priori probability of the occurrence of an event approaches zero in a specified way. Given the personal accuracy of observation of an individual observer the probability of the occurrence of an event to which he attests can be determined for any case of a rather general type, and conversely. The results are then extended to the case of $n$ observers. Finally, a method is obtained of determining the personal accuracy of observation of an individual observer to any degree of approximation, thus furnishing a means of obtaining the data upon which the foregoing results depend.

33. Professor C. H. Forsyth: The determination of how much one would be justified in expending to extend the life and multiply the value of an article.

Students of the mathematical theory of finance are familiar with the problem of determining how much one would be justified in expending to extend the life of an article a specified period, and the corresponding formula. In this paper the problem is extended and a formula derived for 
determining how much one would be justified in expending to extend the life and at the same time multiply the value or productivity of the article. In fact, two formulas are derived so that a criterion of the extra expense can be determined whether both the life and the value of the article are increased or only one (either one) is increased and the other decreased.

34. Dr. Norbert Wiener: A contribution to the theory of interpolation.

Given a finite set of points $S$, surrounded by a curve $C$, the properties are developed of that function, harmonic on the interior of $C$, which assumes given values over $S$ and has the least integral around $C$.

35. Professor L. R. Ford: The numerical integration of differential equations.

In solving a differential equation by Picard's method of successive approximations, the repeated integrations can be performed by the approximate methods of finite differences or by the use of a few terms of the Euler-Maclaurin expansion. Two questions arise: Does the numerical process converge? If so, what are the errors of the approximate solution? In this paper, it is proved that the process converges under quite general conditions, and a limit is found for the errors that result when the approximate method is repeated over successive intervals.

36. Mr. A. D. Michal: Invariant functionals of closed plane curves under one-parameter groups of transformations of the plane.

In this paper the author shows that there always exist functionals of closed plane curves invariant under a given arbitrary continuous one-parameter group of transformations of the plane. The functional of closed plane curves is assumed to be developable in a new type of expansion involving multiple double integrals, the region of integration being that of the area of the closed curves. The necessary and sufficient conditions for the invariance of the functionals in question reduce to the form of certain non-homogeneous linear partial differential equations of the first order. The symmetric solutions of these partial differential equations 
yield the sought for integrands found in the assumed expansion of the invariant functionals in question. The actual calculation of the invariants is effected completely in the case of a number of well known continuous one-parameter groups of transformations of the plane.

37. Professor J. F. Ritt: Note on Dirichlet series with complex exponents.

This note gives a very simple proof of an extension made by E. Hille of a theorem of the present author's.

38. Professor E. L. Dodd: Integration in certain n-dimensional fields with the aid of a general discontinuity factor.

Use is made of $F(t)$, where $F(0)=0$ and

$$
\int_{-\infty}^{\infty} \frac{F(t) d t}{t}=K \neq 0, \neq \infty,
$$

to effect integration of real functions in fields such as that determined by $-a<X<0$, where $X$ is a real function defined for all real values of its $n$ variables. The general formula thus obtained, when applied to the case of $n=1$, with $F(t)=\sin t$, and $X$ linear, yields the Fourier integral equation. The discontinuity-factor method, used in the theory of probability to integrate $\varphi_{1}\left(x_{1}\right) \varphi_{2}(x) \ldots \varphi_{n}\left(x_{n}\right)$ between parallel planes, is shown to be valid in case each $\varphi_{i}\left(x_{i}\right)$ is bounded and integrable and absolutely integrable, and two of these functions are of limited variation. This device of integration, which makes integration proceed to $\infty$, while keeping the $n$ variables separate, may then be used for almost any bounded function $\varphi_{i}\left(x_{i}\right)$ that has been suggested for frequency distributions, such as the Pearson types, finite Brun's series, Poisson exponential function, Charlier B-series, Jorgensen function, and Makeham life function.

R. G. D. RICHARDSON, Secretary. 